\title{
Vulnerabilidade de mulheres que fazem sexo com mulheres às infecções sexualmente transmissíveis
}

\author{
Vulnerability to sexually transmitted infections of women \\ who have sex with women
}

Juliane Andrade (http://orcid.org/0000-0002-4321-0118) ${ }^{1}$

Mariana Alice Oliveira Ignácio (http://orcid.org/0000-0002-3209-035X) ${ }^{2}$

Ana Paula Freneda de Freitas (http://orcid.org/0000-0002-3796-6111) ${ }^{3}$

Cristina Maria Garcia de Lima Parada (https://orcid.org/0000-0002-9597-3635) ${ }^{4}$

Marli Teresinha Cassamassimo Duarte (http://orcid.org/0000-0001-9582-2944) ${ }^{4}$

${ }^{1}$ Departamento de Enfermagem, Faculdade de Ciências da Saúde, Universidade de Brasília. Campus Universitário Darcy Ribeiro s/n, Asa Norte. 70910-900 Brasília DF Brasil.juenf_andrade@ yahoo.com.br

${ }^{2}$ Departamento de Saúde Pública, Faculdade de Medicina de Botucatu, Universidade Estadual Paulista (Unesp). Botucatu SP Brasil.

${ }^{3}$ Centro de Saúde Escola, Unesp. Botucatu SP Brasil.

${ }^{4}$ Departamento de Enfermagem, Faculdade de Medicina de Botucatu, Unesp. Botucatu SP Brasil.
Abstract The scope of this study was to assess the degree of vulnerability to sexually transmitted infections of women who have sex with women. It involved a cross-sectional study of 150 women between 2015 and 2017. A structured questionnaire was applied, and a gynecological examination was performed to diagnose Chlamydia trachomatis, Neisseria gonorrhoeae, Trichomonas vaginalis and papillomavirus. Blood tests were conducted to detect HIV, hepatitis B and syphilis. The outcome variable was sexual infection and the independent variables comprised the vulnerability level in the individual, social and programmatic dimensions. Data analyses were performed through logistic regression. The results showed a high prevalence of infections (47.3\%) and only variables of individual vulnerability were associated with the outcome. The incidence of infection was four times higher among women who had had prior infections. The fact of never having had a blood test tripled the chance of having sexually transmitted infections. The fact of also having sexual intercourse with men in the previous 12-month-period increased the risk of the outcome by a factor of approximately nine. The conclusion drawn is that these women are vulnerable to sexually transmitted infections due to their individual vulnerability.

Key words Vulnerability, Sexually transmitted infections, Female homosexuality
Resumo O objetivo do presente estudo foi identificar as dimensões da vulnerabilidade de mulheres que fazem sexo com mulheres associadas às infecções sexualmente transmissiveis. Estudo transversal com 150 mulheres, entre 2015-2017. Aplicouse questionário estruturado, realizou-se exame ginecológico para diagnóstico de Chlamydia trachomatis, Neisseria gonorrhoeae, Trichomonas vaginalis, papiloma vírus humano e coleta de sangue periférico para detecção de HIV, hepatite $B e$ sifilis. A variável desfecho foi a ocorrência de infecção sexual e as variáveis independentes compõem o referencial da vulnerabilidade, nas dimensões individual, social e programática. Análise de dados foi realizada por modelo de regressão logística. Os resultados mostraram alta prevalência das infecções $(47,3 \%)$ e apenas variáveis relacionadas à vulnerabilidade individual se associaram de forma independente ao desfecho: chance de infecção foi quatro vezes maior entre mulheres com antecedente de infecção; nunca ter realizado exame sorológico aumentou a chance em quase três vezes e ter tido relação sexual com homem nos últimos 12 meses aumentou em quase nove vezes a chance do desfecho. Concluiu-se que essas mulheres são vulneráveis às infecções sexualmente transmissíveis em decorrência da vulnerabilidade individual.

Palavras-chave Vulnerabilidade, Infecções sexualmente transmissíveis, Homossexualidade feminina 


\section{Introdução}

No Brasil, a Política Nacional de Atenção Integral à Saúde da Mulher (PNAISM, 2004) marca o momento de inclusão das mulheres em sua diversidade, inclusive de orientação sexual, nas políticas públicas de saúde ${ }^{1}$. Essa inclusão é resultado de árdua atuação de movimentos de Lésbicas, Gays, Bissexuais, Travestis e Transexuais (LGBT), militantes lésbicas feministas e de profissionais de saúde, resultando em ações governamentais que estão fazendo o cenário mudar aos poucos².

A vulnerabilidade de mulheres que fazem sexo com mulheres (MSM) perpassa questões como: luta por visibilidade social e política ${ }^{3}$, diferença de gênero ${ }^{4}$, heteronormatividade ${ }^{5}$, despreparo do profissional de saúde para atendimento desse grupo ${ }^{3}$ e o próprio desconhecimento sobre questões relativas à prevenção das Infecções Sexualmente Transmissíveis (IST) ${ }^{6}$.

Nesse sentido, o modelo de vulnerabilidade foi empregado na presente pesquisa, pois esta pondera o coletivo e não o individual e busca a compreensão das exposições de indivíduos ou grupo de indivíduos a dado agravo à saúde por sínteses pragmáticas baseadas em três dimensões analíticas articuladas: a individual, a social e a programática ${ }^{7}$.

A dimensão individual é, fundamentalmente, de ordem cognitiva - qualidade e quantidade de informação de que os indivíduos dispõem, capacidade de elaborá-la e de ordem comportamental - capacidade, habilidade e interesse para transformar essas informações em atitudes e ações protegidas e protetoras ${ }^{7}$. Também está relacionada com as características pessoais como idade, sexo, raça/cor, percepção de risco e conhecimento sobre IST ${ }^{8}$.

A dimensão social envolve o acesso às informações, às possibilidades de metabolizá-las e ao poder de incorporá-las às mudanças práticas na vida cotidiana, condições diretamente associadas ao acesso a recursos materiais (estrutura econômica), a instituições sociais como escolas e serviços de saúde, ao poder de influenciar decisões políticas, à possibilidade de enfrentar barreiras culturais e de estar livre de coerções violentas de todas as ordens ${ }^{7,8}$.

A terceira dimensão, denominada institucional ou programática, conecta os componentes individual e social. Envolve o grau e a qualidade de compromisso, recursos, gerência e monitoramento de programas nacionais, regionais ou locais de prevenção e cuidado, os quais são importantes para identificar necessidades, canalizar os recursos sociais existentes e otimizar seu uso? ${ }^{7}$.

Considerando a escassez de estudos brasileiros sobre a saúde de MSM e que, mesmo no contexto internacional, o referencial teórico da vulnerabilidade não é empregado quando se aborda essa temática e considerando ainda que a ampliação de estudos na área poderá aprimorar o conhecimento das necessidades da saúde sexual e reprodutiva dessas mulheres e, consequentemente, qualificar o cuidado, propôs-se o presente estudo a identificar as variáveis das dimensões da vulnerabilidade de MSM associadas às IST.

A designação MSM foi adotada neste estudo tendo em vista que práticas sexuais entre mulheres são diversas durante a vida, independente da orientação e identidade sexual ${ }^{9}$ : a referência a uma identidade sexual (homossexual, gay, travesti) não necessariamente traduz a prática sexual com uma pessoa do mesmo sexo, enquanto a definição heterossexual não exclui a prática sexual com pessoas do mesmo sexo ${ }^{10}$. Por isso, recomenda-se a essa população ações de prevenção às IST/ aids que priorizem a história sexual, motivo pelo qual o presente artigo utiliza-se da sigla MSMํ.

\section{Método}

Foi realizado estudo transversal que compõe pesquisa mais ampla denominada "Acesso a serviços de saúde e saúde sexual e reprodutiva de mulheres que fazem sexo com mulheres" ${ }^{\prime 1}$, desenvolvido no município de Botucatu, São Paulo (SP), interior paulista, com suporte financeiro da Fundação de Amparo à Pesquisa do Estado de São Paulo.

A população-alvo do estudo foi constituída por mulheres que declararam fazer sexo com mulheres ou com mulheres e com homens, acima de 18 anos, residentes em municípios pertencentes ao Departamento Regional de Saúde de Bauru (DRS -VI), Polos Cuesta, Vale do Jurumirim, Bauru e Jaú. Foram critérios de exclusão: recusa em participar das três etapas da pesquisa (resposta ao questionário, realização de exame ginecológico e coleta de sangue periférico) e amostra cervical inadequada para realização do diagnóstico laboratorial de IST.

Para recrutamento das participantes, realizou-se a divulgação do projeto nas redes de sociabilidade (página do Facebook por cartazes e panfletos), comunicação de massa (rádio, televisão e jornal impresso), serviços de saúde (campanhas, palestras e reuniões de colegiado de gestores municipais), indicação de profissionais 
de saúde, liderança LGBT e das próprias MSM participantes. Nesse último caso, aplicando-se a técnica de amostragem Bola de $\mathrm{Neve}^{12}$, de posse dos contatos, as pesquisadoras realizavam o convite por telefone, expondo o objetivo da pesquisa, a forma de participação, a garantia de privacidade e agendando a data, horário e local da coleta de dados.

Apenas duas mulheres procuraram espontaneamente as pesquisadoras, 46 foram indicadas por profissionais da saúde, 19 por lideranças LGBT e 256 por participantes do estudo. Dessa forma, 323 MSM foram identificadas, mas apenas 293 foram localizadas, dentre as quais 35 recusaram-se a participar do estudo e 18 não atenderam aos critérios de inclusão, totalizando 240 mulheres. Dessas, 60 não compareceram e 30 foram excluídas por não aceitarem fazer o exame ginecológico (24 casos) ou em decorrência da inadequação da amostra para a realização de diagnóstico laboratorial de IST (seis casos). Assim, a amostra foi constituída por 150 MSM e o detalhamento de sua composição encontra-se no diagrama de constituição da amostra exposto por Ignácio et al. ${ }^{13}$.

Os dados foram obtidos de janeiro de 2015 a abril de 2017, por meio de instrumento_construído especificamente para o presente estudo, com base na literatura correlata à temática e submetido à análise de profissionais especialistas na área, inclusive uma profissional de saúde que faz sexo com mulheres, quanto à sua clareza e conteúdo. Houve, além disso, teste prévio com uma MSM que não integrou a amostra do estudo.

A coleta de dados ocorreu em três etapas: aplicação de questionário, coleta de sangue periférico e realização de exame ginecológico, nessa ordem e no mesmo dia. Foi realizada no serviço de saúde onde funciona o Programa Municipal de DST/ aids pelas três primeiras autoras deste estudo, após capacitação. O ambiente era confortável e privativo e o tempo médio de coleta foi 40 minutos.

A variável dependente ou desfecho foi ter o diagnóstico de alguma IST: Chlamydia trachomatis, Neisseria gonorrhoeae, Trichomonas vaginalis, infecções por Papiloma Vírus Humano (HPV), Vírus da Imunodeficiência Humana (HIV), hepatite B ou sífilis. Para identificar as variáveis independentes nas dimensões da vulnerabilidade, adotou-se, como referencial teórico, o quadro vulnerabilidade e direitos humanos: dimensões individual, social e programática proposto por Ayres et al. ${ }^{10}$.

A dimensão individual foi composta pelas seguintes variáveis dicotômicas: idade $\leq 24$ anos, cor da pele não branca (autodeclarada), escolaridade $<$ que 8 anos, união estável, frequenta serviços de saúde para ações de prevenção, conhecimentos básicos sobre IST, antecedente de IST, sorologias para IST, percepção de risco para IST, percepção de risco para HIV, sexo após consumo de droga ilícita e/ou bebida alcoólica, idade da coitarca $\leq 14$ anos, parceria eventual nos últimos 12 meses, duas ou mais parcerias sexuais nos últimos 12 meses, última parceira teve sexo com homem, histórico de prática sexual em troca de dinheiro e/ou drogas, recebe penetração vaginal, recebe penetração anal, uso inconsistente de preservativo, pratica tribadismo, relação sexual no período menstrual, alteração na microbiota vaginal, relação sexual com homem na vida e relação sexual com homem nos últimos 12 meses.

A dimensão social constituiu-se por: ter atividade remunerada, renda per capita $<\mathrm{R} \$ 291,00^{14}$, referir ao serviço de saúde que faz sexo com mulher, ser vítima de preconceito e abuso sexual na vida. A dimensão Programática compreendeu: frequentar o serviço de Atenção Primária à Saúde, disponibilizada citologia oncótica (CO), receber informações sobre IST nos serviços de saúde, apresentar dificuldade de acesso aos serviços de saúde bem como dificuldade na relação com o profissional de saúde.

Para o diagnóstico das infecções pelo HPV, Chlamydia trachomatis e Neisseria gonorrhoeae empregou-se a reação em cadeia da polimerase; para confirmação do HIV, os testes ELISA e Western Blot; para a Hepatite B, o ensaio ARCHITECT HBsAg; para a sífilis a presença de teste treponêmico e VDRL reagentes ou dois treponêmicos distintos positivos, sem história de tratamento prévio adequado e a identificação do Trichomonas vaginalis se deu por meio da coloração de Papanicolaou.

Os dados foram analisados por estatística descritiva e a associação entre as variáveis de vulnerabilidade e o diagnóstico de IST foi realizada por meio de modelo de regressão logística simples. Em seguida, as variáveis que mais influenciaram no desfecho $(\mathrm{p}<0,20)$ foram levadas para o modelo de regressão logística múltiplo, no qual se identificaram aquelas independentemente associadas ao desfecho $(\mathrm{p}<0,05)$.

$O$ projeto desta pesquisa foi submetido e avaliado pelo Comitê de Ética em Pesquisa (CEP) da Faculdade de Medicina de Botucatu da Universidade Estadual Paulista "Júlio de Mesquita Filho". Todas as participantes deste estudo foram devidamente esclarecidas sobre os objetivos e forma de participação e, para aquelas que aceitaram, 
foi solicitado assinar o Termo de Consentimento Livre e Esclarecido em duas vias, ficando uma cópia com as pesquisadoras e a outra, com a participante. As mulheres que tiveram resultados de exames alterados foram contatadas, orientadas e encaminhadas para o ambulatório de referência para tratamento.

\section{Resultados}

O diagnóstico de alguma IST foi constatado em 71 mulheres $(47,3 \%)$. A prevalência da infecção pelo HPV foi de $45,3 \%$, Chlamydia trachomatis de 2,0\%, pelo HIV e Neisseria gonorrhoea de $0,7 \%$ e tricomoníase e sífilis foram detectadas em 1,3\% das MSM investigadas. A infecção pelo vírus da Hepatite B não foi identificada.

Considerando-se a dimensão individual da vulnerabilidade, a maioria das MSM estudadas não tinha união estável $(73,3 \%)$, não se percebia em risco de IST e de infecção pelo HIV (56,7\% e 67,3\%, respectivamente), tinha relação sexual após uso de drogas ilícitas e/ou álcool (71,3\%), recebia penetração vaginal $(88,0 \%)$, não fazia uso consistente do preservativo $(82,0 \%)$ e tinha história de relação sexual com homem na vida $(74,7 \%)$ (Tabela 1$)$.

Parte das mulheres tinha idade igual ou inferior a 24 anos $(42,7 \%)$, não procuravam os serviços de saúde para ações de prevenção (42,7\%), não tinham realizado sorologia para diagnóstico de IST $(46,7 \%)$, haviam tido duas ou mais parcerias no último ano $(40,7 \%)$, sua última parceira havia tido relação sexual com homem $(45,3 \%)$ e apresentavam alteração na microbiota vaginal $(46,7 \%)$. Destaca-se, ainda, que um quarto das mulheres $(25,3 \%)$ tinha cor da pele não branca, $19,3 \%$ não tinham conhecimentos básicos sobre IST, 10,7\% tinham antecedente de IST, $22,0 \%$ praticavam tribadismo, 24,7\% tinham relação no período menstrual e $21,3 \%$ haviam tido relação sexual com homem nos últimos 12 meses (Tabela 1).

A análise univariada apontou que as variáveis de vulnerabilidade individual mais fortemente associadas com o diagnóstico laboratorial de IST foram: idade igual ou inferior a 24 anos, cor da pele não branca, união estável, conhecimentos básicos sobre IST, antecedente de IST, sorologia para IST, percepção de risco de IST e de HIV, parceria eventual, duas ou mais parcerias nos últimos 12 meses, recebe penetração vaginal e anal, relação sexual no período menstrual, relação sexual com homem na vida e nos últimos 12 meses (Tabela 1).
No que se refere à vulnerabilidade social, a maioria das mulheres $(58,0 \%)$ tinha sido vítima de preconceito e $47,3 \%$ não revelavam aos profissionais de saúde fazer sexo com mulher, 26,0\% não tinham atividade remunerada e $15,3 \%$ tinham sofrido abuso sexual (Tabela 2). Foram selecionadas, como potencialmente associadas com IST, as seguintes variáveis da dimensão social: atividade remunerada e renda per capta abaixo de $\mathrm{R} \$ 291,00$ (Tabela 2).

No contexto da vulnerabilidade programática, a maioria das MSM não recebeu informações sobre IST nos serviços de saúde $(83,3 \%)$, pouco mais da metade $(50,7 \%)$ referiram dificuldade de acesso a estes, relacionada a sua estrutura e organização e 33,3\% delas não frequentavam serviços de Atenção Primária à Saúde (Tabela 2). Variáveis da dimensão programática não foram associadas às IST na análise univariada.

$\mathrm{Na}$ análise de regressão logística multivariada, apenas as variáveis da dimensão individual da vulnerabilidade, quais sejam, antecedente de IST, sorologia para IST e relação sexual com homem nos últimos 12 meses foram independentemente associadas ao diagnóstico de IST confirmado por exames laboratoriais. A chance de diagnóstico de IST foi quatro vezes maior entre mulheres com antecedente para IST do que entre aquelas sem esse histórico [OR = 4,00 (1,03-15,50); p = $0,045]$. Nunca ter realizado sorologia para IST aumentou a chance em quase três vezes de resultado confirmado dessas infecções [OR $=2,80$ $(1,13-6,94) ; \mathrm{p}=0,027]$ e haver tido relação sexual com homem nos últimos 12 meses aumentou em quase nove vezes a chance do desfecho [OR = $8,65(2,39-31,38) ; \mathrm{p}=0,001]$ (Tabela 3).

\section{Discussão}

A presente pesquisa permitiu identificar as dimensões de vulnerabilidade às IST de MSM e as variáveis das dimensões da vulnerabilidade que se associaram ao diagnóstico confirmado por exames laboratoriais dessas infecções, possibilitando, assim, a sinalização de aspectos prioritários para a intervenção.

Ainda que se reconheça que as dimensões da vulnerabilidade se articulam em seus diversos contextos e que seu entrelaçamento dificulta estudos quantitativos/epidemiológicos, devido à possibilidade de uma variável se enquadrar em mais dimensões ${ }^{10}$, optou-se, no presente artigo, por classificá-las em separado, de acordo com o quadro de "vulnerabilidade e direitos humanos: 
Tabela 1. Associação entre diagnóstico de infecção sexualmente transmissível e variáveis de vulnerabilidade da dimensão individual em mulheres que fazem sexo com mulheres $(n=150)$. Botucatu, 2015-2017

\begin{tabular}{|c|c|c|c|c|c|c|c|c|}
\hline \multirow{3}{*}{ Variáveis } & \multicolumn{6}{|c|}{ IST } & \multirow{3}{*}{ OR (IC95\%) } & \multirow{3}{*}{$\mathbf{P}^{\star}$} \\
\hline & \multicolumn{2}{|c|}{ Total } & \multicolumn{2}{|c|}{ Não } & \multicolumn{2}{|c|}{ Sim } & & \\
\hline & $\mathbf{n}$ & $\%$ & $\mathbf{n}$ & $\%$ & $\mathbf{n}$ & $\%$ & & \\
\hline \multicolumn{9}{|l|}{ Vulnerabilidade Individual } \\
\hline \multicolumn{9}{|l|}{ Idade $\leq 24$ anos } \\
\hline Não & 86 & 57,3 & 53 & 61,6 & 33 & 38,4 & 1,0 & - \\
\hline Sim & 64 & 42,7 & 26 & 40,6 & 38 & 59,4 & $2,35(1,21-4,55)$ & 0,011 \\
\hline \multicolumn{9}{|l|}{ Cor da pele não branca } \\
\hline Não & 112 & 74,7 & 64 & 57,1 & 48 & 42,9 & 1,0 & - \\
\hline Sim & 38 & 25,3 & 15 & 39,5 & 23 & 60,5 & $2,04(0,97-4,33)$ & 0,062 \\
\hline \multicolumn{9}{|l|}{ Escolaridade $<$ que 8 anos } \\
\hline Não & 144 & 96,0 & 75 & 52,1 & 69 & 47,9 & 1,0 & - \\
\hline Sim & 6 & 4,0 & 4 & 66,7 & 2 & 33,3 & $0,54(0,10-3,06)$ & 0,489 \\
\hline \multicolumn{9}{|l|}{ União estável } \\
\hline Não & 110 & 73,3 & 50 & 45,5 & 60 & 54,5 & $3,16(1,44-6,96)$ & 0,004 \\
\hline Sim & 40 & 26,7 & 29 & 72,5 & 11 & 27,5 & 1,0 & - \\
\hline \multicolumn{9}{|c|}{$\begin{array}{l}\text { Frequenta serviços de saúde para ações de } \\
\text { prevenção }\end{array}$} \\
\hline Não & 64 & 42,7 & 30 & 46,9 & 34 & 53,1 & $1,50(0,78-2,88)$ & 0,221 \\
\hline Sim & 86 & 57,3 & 49 & 57,0 & 37 & 43,0 & 1,0 & - \\
\hline \multicolumn{9}{|c|}{ Conhecimentos básicos sobre IST ${ }^{\S}$} \\
\hline Não & 29 & 19,3 & 12 & 41,4 & 17 & 58,6 & $1,76(0,77-4,00)$ & 0,178 \\
\hline Sim & 121 & 80,7 & 67 & 55,4 & 54 & 44,6 & 1,0 & - \\
\hline \multicolumn{9}{|l|}{ Antecedente de IST ${ }^{\S}$} \\
\hline Não & 134 & 89,4 & 74 & 55,2 & 60 & 44,8 & 1,0 & - \\
\hline $\operatorname{Sim}$ & 16 & 10,6 & 5 & 31,3 & 11 & 68,8 & $2,71(0,89-8,24)$ & 0,078 \\
\hline \multicolumn{9}{|l|}{ Sorologias para IST ${ }^{\S}$} \\
\hline Não & 70 & 46,7 & 31 & 44,3 & 39 & 55,7 & $1,89(0,99-3,62)$ & 0,056 \\
\hline Sim & 80 & 53,3 & 48 & 60,0 & 32 & 40,0 & 1,0 & - \\
\hline \multicolumn{9}{|l|}{ Percepção de risco para IST ${ }^{\S}$} \\
\hline Não & 85 & 56,7 & 50 & 58,8 & 35 & 41,2 & $0,56(0,29-1,08)$ & 0,085 \\
\hline Sim & 65 & 43,3 & 29 & 44,6 & 36 & 55,4 & 1,0 & - \\
\hline \multicolumn{9}{|l|}{ Percepção de risco para HIV } \\
\hline Não & 101 & 67,3 & 58 & 57,4 & 43 & 42,6 & $0,56(0,28-1,11)$ & 0,095 \\
\hline Sim & 49 & 32,7 & 21 & 42,9 & 28 & 57,1 & 1,0 & - \\
\hline \multicolumn{9}{|c|}{$\begin{array}{l}\text { Sexo após consumo de droga ilícita e/ou } \\
\text { bebida alcoólica }\end{array}$} \\
\hline Não & 43 & 28,7 & 26 & 60,5 & 17 & 39,5 & 1,0 & - \\
\hline Sim & 107 & 71,3 & 53 & 49,5 & 54 & 50,5 & $1,56(0,76-3,20)$ & 0,227 \\
\hline \multicolumn{9}{|l|}{ Idade da coitarca $\leq 14$ anos } \\
\hline Não & 114 & 76,0 & 60 & 52,6 & 54 & 47,4 & 1,0 & - \\
\hline Sim & 36 & 24,0 & 19 & 52,8 & 17 & 47,2 & $0,99(0,47-2,11)$ & 0,988 \\
\hline \multicolumn{9}{|c|}{ Parceria eventual nos últimos 12 meses } \\
\hline Não & 107 & 71,3 & 61 & 57,0 & 46 & 43,0 & 1,0 & - \\
\hline Sim & 43 & 28,7 & 18 & 41,9 & 25 & 58,1 & $1,84(0,90-3,77)$ & 0,095 \\
\hline \multicolumn{9}{|c|}{$\begin{array}{l}\text { Duas ou mais parcerias sexuais nos últimos } \\
12 \text { meses }\end{array}$} \\
\hline Não & 89 & 59,3 & 54 & 60,7 & 35 & 39,3 & 1,0 & - \\
\hline Sim & 61 & 40,7 & 25 & 41,0 & 36 & 59,0 & $2,22(1,14-4,32)$ & 0,019 \\
\hline
\end{tabular}


Tabela 1. Associação entre diagnóstico de infecção sexualmente transmissível e variáveis de vulnerabilidade da dimensão individual em mulheres que fazem sexo com mulheres $(n=150)$. Botucatu, 2015-2017

\begin{tabular}{|c|c|c|c|c|c|c|c|c|}
\hline \multirow{3}{*}{ Variáveis } & \multicolumn{6}{|c|}{ IST } & \multirow{3}{*}{ OR (IC95\%) } & \multirow{3}{*}{$\mathbf{P}^{\star}$} \\
\hline & \multicolumn{2}{|c|}{ Total } & \multicolumn{2}{|c|}{ Não } & \multicolumn{2}{|c|}{ Sim } & & \\
\hline & $\mathbf{n}$ & $\%$ & n & $\%$ & $\mathbf{n}$ & $\%$ & & \\
\hline \multicolumn{9}{|c|}{ Última parceira teve sexo com homem } \\
\hline Não & 82 & 54,7 & 41 & 50,0 & 41 & 50,0 & 1,0 & - \\
\hline Sim & 68 & 45,3 & 38 & 55,9 & 30 & 44,1 & $0,79(0,41-1,51)$ & 0,473 \\
\hline \multicolumn{9}{|c|}{$\begin{array}{l}\text { Histórico de prática sexual em troca de } \\
\text { dinheiro e/ou drogas }\end{array}$} \\
\hline Não & 147 & 98,0 & 78 & 53,1 & 69 & 46,9 & 1,0 & - \\
\hline Sim & 3 & 2,0 & 1 & 33,3 & 2 & 66,7 & $2,26(0,20-25,48)$ & 0,509 \\
\hline \multicolumn{9}{|l|}{ Recebe penetração vaginal } \\
\hline Não & 18 & 12,0 & 13 & 72,2 & 5 & 27,8 & 1,0 & - \\
\hline Sim & 132 & 88,0 & 66 & 50,0 & 66 & 50,0 & $2,60(0,88-7,71)$ & 0,085 \\
\hline \multicolumn{9}{|l|}{ Recebe penetração anal } \\
\hline Não & 121 & 80,7 & 68 & 56,2 & 53 & 43,8 & 1,0 & - \\
\hline Sim & 29 & 19,3 & 11 & 37,9 & 18 & 62,1 & $2,10(0,91-4,82)$ & 0,080 \\
\hline \multicolumn{9}{|c|}{ Uso inconsistente de preservativo } \\
\hline Não & 27 & 18,0 & 16 & 59,3 & 11 & 40,7 & 1,0 & - \\
\hline Sim & 123 & 82,0 & 63 & 51,2 & 60 & 48,8 & $1,39(0,60-3,23)$ & 0,450 \\
\hline \multicolumn{9}{|l|}{ Pratica tribadismo } \\
\hline Não & 117 & 78,0 & 63 & 53,8 & 54 & 46,2 & 1,0 & - \\
\hline Sim & 33 & 22,0 & 16 & 48,5 & 17 & 51,5 & $1,24(0,57-2,69)$ & 0,586 \\
\hline \multicolumn{9}{|c|}{ Relação sexual no período menstrual } \\
\hline Não & 113 & 75,3 & 63 & 55,8 & 50 & 44,2 & 1,0 & - \\
\hline Sim & 37 & 24,7 & 16 & 43,2 & 21 & 56,8 & $1,65(0,78-3,50)$ & 0,188 \\
\hline \multicolumn{9}{|l|}{ Alteração na microbiota vaginal } \\
\hline Não & 80 & 53,3 & 43 & 53,8 & 37 & 46,3 & 1,0 & - \\
\hline Sim & 70 & 46,7 & 36 & 51,4 & 34 & 48,6 & $1,10(0,58-2,09)$ & 0,776 \\
\hline \multicolumn{9}{|c|}{ Relação sexual com homem na vida } \\
\hline Não & 38 & 25,3 & 26 & 68,4 & 12 & 31,6 & 1,0 & - \\
\hline Sim & 112 & 74,7 & 53 & 47,3 & 59 & 52,7 & $2,41(1,11-5,25)$ & 0,027 \\
\hline \multicolumn{9}{|c|}{$\begin{array}{l}\text { Relação sexual com homem nos últimos } 12 \\
\text { meses }\end{array}$} \\
\hline Não & 118 & 78,7 & 73 & 61,9 & 45 & 38,1 & 1,0 & - \\
\hline Sim & 32 & 21,3 & 6 & 18,8 & 26 & 81,3 & $7,03(2,69-18,40)$ & 0,000 \\
\hline
\end{tabular}

dimensões individual, social e programática”, proposto por Ayres et al. ${ }^{10}$.

Dentre as variáveis investigadas, apenas as classificadas na dimensão individual foram independentemente associadas ao diagnóstico de IST: antecedente de IST, realização de sorologia para IST e relação sexual com homem nos últimos 12 meses.

O percentual de antecedente de IST referido pelas MSM incluídas na presente investigação $(10,6 \%)$ foi inferior ao obtido tanto em única pesquisa nacional publicada $(38,6 \%)^{15}$ quanto em investigações internacionais $\left(17,0 \%{ }^{16} \mathrm{e}\right.$ $44,0 \%{ }^{17}$ ). Tal fato pode estar relacionado à baixa realização de sorologias como também à não utilização dos serviços de saúde para ações de prevenção e promoção, que possibilitariam seu rastreio entre as MSM participantes.

Históricos de IST e de realização de sorologia para IST não foram investigados em estudos que objetivaram identificar fatores associados às IST em MSM ${ }^{18,19}$, apesar do diagnóstico prévio de IST 
Tabela 2. Associação entre diagnóstico de infecção sexualmente transmissível e variáveis de vulnerabilidade das dimensões social e programática em mulheres que fazem sexo com mulheres $(n=150)$. Botucatu, 2015-2017

\begin{tabular}{|c|c|c|c|c|c|c|c|c|}
\hline \multirow{3}{*}{ Variáveis } & \multicolumn{6}{|c|}{ IST } & \multirow{3}{*}{ OR (IC95\%) } & \multirow{3}{*}{$\mathbf{P}^{*}$} \\
\hline & \multicolumn{2}{|c|}{ Total } & \multicolumn{2}{|c|}{ Não } & \multicolumn{2}{|c|}{ Sim } & & \\
\hline & n & $\%$ & $\mathbf{n}$ & $\%$ & $\mathbf{n}$ & $\%$ & & \\
\hline \multicolumn{9}{|l|}{ Vulnerabilidade Social } \\
\hline \multicolumn{9}{|l|}{ Atividade remunerada } \\
\hline Não & 39 & 26 & 16 & 41,0 & 23 & 59,0 & $1,89(0,90-3,96)$ & 0,093 \\
\hline $\operatorname{Sim}$ & 111 & 74 & 63 & 56,8 & 48 & 43,2 & 1,0 & - \\
\hline \multicolumn{9}{|l|}{ Renda per capita $<\mathrm{R} \$ 291,00$} \\
\hline Não & 5 & 3,3 & 1 & 20,0 & 4 & 80,0 & $4,66(0,51-42,68)$ & 0,174 \\
\hline Sim & 145 & 96,7 & 78 & 53,8 & 67 & 46,2 & 1,0 & - \\
\hline \multicolumn{9}{|c|}{$\begin{array}{l}\text { Referir ao serviço de saúde que faz sexo com } \\
\text { mulher }\end{array}$} \\
\hline Não & 71 & 47,3 & 36 & 50,7 & 35 & 49,3 & $1,16(0,61-2,21)$ & 0,648 \\
\hline Sim & 79 & 52,7 & 43 & 54,4 & 36 & 45,6 & 1,0 & - \\
\hline \multicolumn{9}{|l|}{ Vítima de preconceito } \\
\hline Não & 63 & 42 & 33 & 52,4 & 30 & 47,6 & 1,0 & - \\
\hline Sim & 87 & 58 & 46 & 52,9 & 41 & 47,1 & $0,98(0,51-1,88)$ & 0,952 \\
\hline \multicolumn{9}{|l|}{ Abuso sexual na vida } \\
\hline Não & 127 & 84,7 & 69 & 54,3 & 58 & 45,7 & 1,0 & - \\
\hline Sim & 23 & 15,3 & 10 & 43,5 & 13 & 56,5 & $1,55(0,63-3,79)$ & 0,340 \\
\hline \multicolumn{9}{|l|}{ Vulnerabilidade Programática } \\
\hline \multicolumn{9}{|c|}{ Frequenta o serviço de Atenção Primária à } \\
\hline \multicolumn{9}{|l|}{ Saúde } \\
\hline Não & 50 & 33,3 & 25 & 50,0 & 25 & 50,0 & $1,17(0,60-2,32)$ & 0,644 \\
\hline Sim & 100 & 66,7 & 54 & 54,0 & 46 & 46,0 & 1,0 & - \\
\hline \multicolumn{9}{|c|}{ Disponibilizado citologia oncótica } \\
\hline Não & 13 & 8,7 & 9 & 69,2 & 4 & 30,8 & $0,46(0,14-1,58)$ & 0,219 \\
\hline Sim & 137 & 91,3 & 70 & 51,1 & 67 & 48,9 & 1,0 & - \\
\hline \multicolumn{9}{|c|}{$\begin{array}{l}\text { Recebeu informações sobre IST\$ nos serviços } \\
\text { de saúde }\end{array}$} \\
\hline Não & 125 & 83,3 & 66 & 52,8 & 59 & 47,2 & $0,97(0,41-2,29)$ & 0,942 \\
\hline $\operatorname{Sim}$ & 25 & 16,7 & 13 & 52,0 & 12 & 48,0 & 1,0 & - \\
\hline \multicolumn{9}{|c|}{ Dificuldade de acesso aos serviços de saúde ${ }^{\dagger}$} \\
\hline Não & 74 & 49,3 & 37 & 50,0 & 37 & 50,0 & 1,0 & - \\
\hline Sim & 76 & 50,7 & 42 & 55,3 & 34 & 44,7 & $0,81(0,43-1,54)$ & 0,519 \\
\hline \multicolumn{9}{|c|}{$\begin{array}{l}\text { Dificuldade na relação com o profissional de } \\
\text { saúde }{ }^{\ddagger}\end{array}$} \\
\hline Não & 144 & 96 & 75 & 52,1 & 69 & 47,9 & 1,0 & - \\
\hline $\operatorname{Sim}$ & 6 & 4 & 4 & 66,7 & 2 & 33,3 & $0,54(0,10-3,06)$ & 0,489 \\
\hline
\end{tabular}

sugerir vulnerabilidade das mulheres em outros momentos da vida ${ }^{20}$.

As mulheres que não haviam realizado sorologia para IST anteriormente tiveram chance aumentada em quase três vezes de ter uma IST, o que pode ser explicado em função de que o rastreio viabiliza o diagnóstico e o tratamento. Nesse sentido, destaca-se a íntima relação dessa vari- ável, classificada na dimensão individual, com a vulnerabilidade programática.

Dessa forma, esse dado chamou a atenção, visto que parcela significativa de mulheres não tinha realizado sorologia para diagnóstico de IST: quase metade das MSM estudadas. Tal fato pode ser explicado em função de que um percentual significativo de mulheres $(42,7 \%)$ não frequenta- 
Tabela 3. Modelo de regressão logística múltipla entre variáveis de vulnerabilidade nas dimensões individual, social e diagnóstico de infecções sexualmente transmissíveis em mulheres que fazem sexo com mulheres ( $\mathrm{n}=$ 150). Botucatu, 2015-2017.

\begin{tabular}{lcc}
\hline \multicolumn{1}{c}{ Variáveis } & OR ajustado (IC95\%) & p \\
\hline Vulnerabilidade Individual & & 0,287 \\
Idade $\leq 24$ anos & $1,66(0,65-4,23)$ & 0,120 \\
Cor da pele não branca & $2,10(0,82-5,34)$ & 0,106 \\
União estável & $2,27(0,84-6,17)$ & 0,103 \\
Conhecimentos básicos sobre ISTS & $2,41(0,84-6,90)$ & 0,045 \\
Antecedente de IST § & $4,00(1,03-15,50)$ & 0,027 \\
Sorologias para ISTS & $2,80(1,13-6,94)$ & 0,809 \\
Percepção de risco para IST § & $0,86(0,26-2,83)$ & 0,572 \\
Percepção de risco para HIV & $0,70(0,20-2,45)$ & 0,483 \\
Parceria eventual nos últimos 12 meses & $0,63(0,18-2,26)$ & 0,787 \\
Duas ou mais parcerias sexuais nos últimos 12 meses & $0,85(0,25-2,82)$ & 0,370 \\
Recebe penetração vaginal & $1,91(0,46-7,91)$ & 0,505 \\
Recebe penetração anal & $1,44(0,49-4,22)$ & 0,732 \\
Relação sexual no período menstrual & $1,19(0,44-3,17)$ & 0,293 \\
Relação sexual com homem na vida & $1,70(0,63-4,56)$ & 0,001 \\
Relação sexual com homem nos últimos 12 meses & $8,65(2,39-31,38)$ & \\
Vulnerabilidade Social & & 0,865 \\
Atividade remunerada & $1,09(0,41-2,88)$ & 0,606 \\
Renda per-capita< R\$ 291,00 & $1,94(0,16-23,76)$ & \\
\hline
\end{tabular}

${ }^{\S}$ IST: Infecção Sexualmente Transmissível.

va os serviços de saúde para ações de prevenção. Em pesquisa com mulheres brasileiras vivendo com HIV, constatou-se que elas realizavam mais sorologias quando eram solicitadas por um profissional de saúde $(30 \%)$ do que por iniciativa própria $(12,7 \%)^{21}$.

Entretanto, há que se considerar que a realização de campanhas para o aumento da oferta de sorologias para diagnóstico da infecção pelo HIV e sífilis vem sendo estimulada no Brasil em diversas oportunidades, como, por exemplo, no carnaval e em comemoração ao dia de luta conta a aids ( $1^{\circ}$ de dezembro $)^{22}$. No Estado de São Paulo, a campanha de oferta de exames iniciou em 2008 com sorologia convencional para o HIV e, a partir de 2012, introduziu-se o teste rápido para HIV e sífilis ${ }^{23}$. A ampliação do acesso à testagem permite o diagnóstico precoce dessas infecções, acesso ao tratamento, com o objetivo de cura no caso da sífilis e controle da infecção no caso do HIV, além de proporcionar a quebra da cadeia de transmissão $0^{23}$.

A falta de percepção de risco de MSM para IST e HIV identificada no presente estudo $(56,7 \%$ e $67,3 \%$, respectivamente) também foi observada em estudos nacional ${ }^{3} \mathrm{e}$ internacional ${ }^{24}$. Esses apontaram que a não percepção de risco se dá em função de que as MSM acreditam que a
IST não é transmitida na relação entre mulheres. Tais dados corroboram resultados de outras pesquisas ${ }^{6,25}$ que mostram a percepção de MSM à vulnerabilidade às IST quando as relações sexuais estão associadas à figura masculina, fato também encontrado nesta pesquisa.

Referente ao preparo dos serviços e profissionais ao cuidado LGBT, a portaria $\mathrm{n}^{\circ} 2.836 \mathrm{de}^{\circ}{ }^{\circ}$ de dezembro de 2011 estabeleceu a Política Nacional de Saúde Integral de LGBT, que tem, por objetivos, dentre outros, instituir mecanismos de gestão para atingir maior equidade no Sistema Único de Saúde (SUS), com especial atenção às demandas e necessidades em saúde da população LGBT $^{26}$. Os pressupostos dessa Política, porém, estão distantes das práticas observadas, visto que os resultados obtidos reforçam o cenário de invisibilidade das MSM nos serviços, implicando o não acolhimento das suas necessidades de saúde. O não acolhimento dessas mulheres nos serviços de saúde e a forma estereotipada de abordá-las são aspectos pesquisados em estudo realizado em Recife, o qual também destacou que, quando as orientam, os profissionais de saúde não o fazem corretamente $^{27}$. O desconhecimento perpassa orientações sobre prevenção de IST, solicitação de exames sorológicos para diagnóstico das IST e realização da citologia oncótica ${ }^{28}$. 
Reforça-se que os profissionais de saúde são influenciados pelas questões socioculturais que são pautadas no princípio da heteronormatividade $^{5}$. Dessa forma, o acolhimento é falho, pois o profissional não se anula de suas convicções pessoais e julgamentos. Assim, é urgente refletir e discutir o acolhimento como instrumento importante do processo de trabalho e na formação e aperfeiçoamento dos profissionais de saúde, de forma a garantir o respeito e os direitos humanos, tanto na rede pública, norteada pelo SUS, que se caracteriza pelos princípios da universalidade de acesso e integralidade do atendimento, quanto na rede privada ${ }^{27}$.

$\mathrm{Na}$ presente pesquisa, metade das MSM investigadas referiu dificuldade de acesso aos serviços de saúde, sendo tal percepção relacionada principalmente a sua estrutura e organização, ressaltando-se que dois terços delas frequentavam os serviços públicos de atenção básica. Esse nível de assistência à saúde tem por objetivo acolher e estabelecer vínculo com a população sob sua responsabilidade e reconhecer suas necessidades de saúde ${ }^{28}$, o que não se observou entre as MSM.

Quanto ao histórico de relação sexual com homem ao longo da vida, estudo realizado por Pinto et al. ${ }^{15}$ em São Paulo apontou percentual de mulheres que tiveram parceiros sexuais ao longo da vida $(76,6 \%)$ muito próximo ao obtido na presente investigação $(74,7 \%)$. Dolan e Davis ${ }^{29}$, em cidade do sudeste dos Estados Unidos, também descreveram percentuais semelhantes aos aqui apresentados: 79,0\% das MSM tiveram relações sexuais com homens durante a vida. Outra pesquisa americana mencionou que apenas $7,0 \%$ das MSM referiram nunca terem feito sexo com homens ${ }^{17}$. Histórico de relação sexual com homens já foi apontado em estudo anterior como fator associado à maior chance de IST em MSM, corroborando os resultados da presente pesquisa ${ }^{30}$.

Enfim, destaca-se o perfil geral das mulheres estudadas, constituído por maioria branca, com alto nível de escolaridade, exercendo atividade remunerada e renda per capita familiar acima de um salário mínimo, o que aponta para condições socioeconômicas favoráveis. Esse aspecto evidencia limitação da presente investigação, pois, mesmo com todas as estratégias de divulgação, não foi possível acessar as mulheres com condições socioeconômicas menos favoráveis, o que possivelmente implicaria perfil de vulnerabilidade diferenciado, especialmente no componente social.

Por outro lado, as mulheres com condições socioeconômicas mais favoráveis podem ter maior suporte para se declararem MSM. Logo, como mencionado anteriormente, essas variáveis não atuam isoladamente e nem da mesma forma para cada indivíduo, pois isso irá depender do contexto em que cada um vive, das relações sociais construídas - comunitárias, familiares e redes de amizades -, que podem influenciar positiva ou negativamente no processo saúde-doença $^{10}$.

Ainda que os dados apresentados não possam ser generalizados, por não se tratar de estudo de base populacional, ressalta-se sua relevância, uma vez que, além de contribuir com o aumento do conhecimento na temática, trata-se do primeiro documento nacional a trabalhar com IST em MSM empregando variáveis na ótica da vulnerabilidade, de acordo com o proposto por Ayres et al. ${ }^{10}$. Para os profissionais da área da saúde, fornecem elementos que facilitam a sistematização da assistência a esse grupo populacional, permitindo intervenções que considerem as três dimensões da vulnerabilidade, implicando maior potencial de transformação do processo-saúdedoença.

\section{Conclusão}

As MSM investigadas apresentavam vulnerabilidade às IST, com ênfase na vulnerabilidade individual, sugerindo a necessidade de atenção individualizada e qualificada com vistas à sua redução, com enfoque especial para aquelas que nunca realizaram sorologia para IST, com histórico de IST e que tiveram relação sexual com homem nos últimos 12 meses.

Sugere-se a realização de outros estudos que abordem a intersubjetividade das dimensões e de cada variável identificada neste estudo como indicativo de vulnerabilidade para a população de MSM. Outras perspectivas relativas às questões da vulnerabilidade social e programática, condizentes às redes sociais e à capacitação de profissionais e instituições de saúde, merecem ser investigadas. 


\section{Colaboradores}

J Andrade: Concepção, planejamento/delineamento, coleta e interpretação dos dados, redação do artigo e revisão, aprovação da versão final e formatação do mesmo. MAO Ignácio: Concepção, coleta e interpretação dos dados, revisão crítica do conteúdo e aprovação da versão final do manuscrito. APF Freitas: Concepção e coleta dos dados e aprovação da versão final do manuscrito. CMGL Parada: interpretação dos dados, revisão crítica do conteúdo e aprovação da versão final do manuscrito. MTC Duarte: Concepção, planejamento/delineamento, interpretação dos dados, redação do artigo e revisão, aprovação da versão final.

\section{Agradecimentos}

À Secretaria Municipal da Saúde de Botucatu e Organização Social Fundação Uni, Espaço Saúde Cecília Magaldi, Programa de DST/ aids de Botucatu e suas equipes pelo acolhimento e comprometimento com a pesquisa. Ao Laboratório de Imunopatologia da Relação Materno-Fetal -FMB pelas análises laboratoriais. A todos os profissionais que se envolveram de alguma forma com a pesquisa $e$ às mulheres que participaram do estudo.

\section{Referências}

1. Brasil. Ministério da Saúde (MS). Política Nacional de Atenção Integral à Saúde da Mulher: princípios e diretrizes. Brasília: MS; 2004.

2. Facchini R, Barbosa RM. Dossiê Saúde das Mulheres Lésbicas - Promoção da equidade e da integralidade. Rede Nacional Feminista de Saúde, Direitos Sexuais e Direitos Reprodutivos_Rede Feminista de Saúde [Internet]. Belo Horizonte, 2006 [acessado 2017 Mar 27]. Disponível em: http://www.academia.edu/2391225/ Dossi\%C3\%AA_Sa\%C3\%BAde_das_Mulheres_L\%C3\%A9sbicas_-_Promo\%C3\%A7\%C3\%A3o_da equidade_e_da_integralidade

3. Barbosa RM, Facchini R. Acesso a cuidados relativos à saúde sexual entre mulheres que fazem sexo com mulheres em São Paulo, Brasil. Cad Saude Publica 2009; 25(Supl. 2):291-300.

4. Facchini R. Histórico da luta de LGBT no Brasil. In: Conselho Regional de Psicologia da 6a Região (CRPSP), organizador. Psicologia e diversidade sexual. São Paulo: CRPSP; 2011. p. 10-19.

5. Valadão RC, Gomes R. A homossexualidade feminina no campo da saúde: da invisibilidade à violência. Physis Rev Saúde Colet 2011; 21(4):1451-1467.

6. Moraes L, Esteves MC. Práticas sexuais de mulheres lésbicas e bissexuais e a relação com a prevenção das DST/HIV/aids. In: Anais do Seminário Internacional Enlaçando Sexualidades; 4-6 Set 2011; Salvador, Brasil. Salvador: Universidade do Estado da Bahia; 2011. p. 1-10.

7. Ayres JRCM, França Júnior I, Calazans GJ, Saletti HCF. O conceito de vulnerabilidade e as práticas de saúde: novas perspectivas e desafios. In: Czeresnia D, Freitas CM, organizadores. Promoção da saúde: conceitos, reflexões, tendências. $2^{\text {a }}$ ed. Rio de Janeiro: Fiocruz; 2009. p. 121-143.

8. Nichiata LYI, Bertilozzi MR, Takahashi RF, Fracolli LA. O conceito de "vulnerabilidade" pela enfermagem. Rev Latino-Am Enfermagem [periódico na Internet]. 2008 Set-Out [acessado 2014 Jan 25]; 16(5): [cerca de 5 p.]. Disponível em: http://www.revistas.usp. br/rlae/article/view/17025/19017

9. Mora C, Monteiro S. Vulnerability to STIs/HIV: sociability and the life trajectories of young women who have sex with women in Rio de Janeiro. Cult Health Sexual 2010; 12(1):115-124.

10. Ayres JR, Paiva V, França Júnior I. Conceitos e práticas de prevenção: da história natural da doença ao quadro da vulnerabilidade e direitos humanos. In: Paiva V, Ayres JR, Buchalla CM. Vulnerabilidade e direitos humanos. Curitiba: Juruá; 2012. p. 71-94.

11. Duarte MTC, Godoy I, Silva MGS, Andrade J, Ignacio MAO, Freitas APF. Acesso a serviços de saúde e saúde sexual e reprodutiva de mulheres que fazem sexo com mulheres [projeto de pesquisa]. Botucatu: Faculdade de Medicina de Botucatu; 2014.

12. Handcock MS, Gile KJ. On the concept of snowball sampling. Sociol Methodol 2011; 41(1):367-371. 
13. Ignacio MAO, Andrade J, Freitas APF, Vitor G, Silva MG, Duarte MTC. Prevalência de vaginose bacteriana e fatores associados em mulheres que fazem sexo com mulheres. Rev. Latino-Am. Enfermagem [periódico na Internet]. 2018 Nov. [acessado $2018 \mathrm{dez} 21$ ]; 26: [cerca de 8p.]. Disponível em: http://www.scielo.br/pdf/ rlae/v26/0104-1169-rlae-26-e3077.pdf.

14. Brasil. Secretaria de Assistência Estudantil. Pergunta e resposta sobre a definição da classe média [Internet]. 2012 [citado 2016 Abr 28]. Disponível em: http:// www.sae.gov.br/wp-content/uploads/Perguntas-e -Respostas-sobre-a-Defini\%C3\%A7\%C3\%A3o-daClasse-M\%C3\%A9dia.pdf

15. Pinto VM, Tancredi MV, Tancredi Neto A, Buchallad CM. Sexually transmitted disease/HIV risk behavior among women who have sex with women. AIDS 2005; 19(Supl. 4):64-69.

16. Diamont AL, Schuster MA, Lever J. Receipt of preventive health care services by lesbians. Am J Prev Med 2000; 19(3):141-148.

17. Fethers K, Marks C, Mindel A. Estcourt CS. Sexually transmitted infections and risk behaviours in women who have sex with women. Sex Transm Infect 2000; 76(5):345-349.

18. Bailey JV, Farquhar C, Owen C, Mangtani P. Sexually transmitted infections in women who have sex with women. Sex Transm Infect 2004; 80(3):244-246.

19. Xiao-Fang W, Norris JL, Ying-Jie L, Reilly KH, Ning W. Health-related attitudes and risk factors for sexually transmitted infections of Chinese women who have sex with women. Chin Med J 2012; 125(16):28192825 .

20. Andrade J, Ayres JA, Alencar RA, Duarte MTC, Parada CMGL. Vulnerabilidade de idosos a infecções sexualmente transmissíveis. Acta Paul Enferm 2017; 30(1):8-15

21. Santos NJS, Barbosa RM, Pinh AA, VillelaWV, Aidar T, Filipe EMV. Contextos de vulnerabilidade para o HIV entre mulheres brasileiras. Cad Saude Publica 2009; 25(Supl. 2):231-333.

22. Brasil. Ministério da Saúde (MS). Campanhas do governo para prevenção [Internet]. Brasília; 2012 [acessado 2017 Mar 27]. Disponível em: http://www.aids. gov.br/campanhas?page $=1$

23. São Paulo. Centro de Referência e Treinamento DST/ AIDS-SP (CRT). Divulgação da $9^{a}$ campanha estadual fique sabendo - 2016: testagem de HIV e sifilis [Internet]. São Paulo; 2016 [acessado 2017 Mar 27]. Disponível em: http://www.saude.sp.gov.br/centrode-referencia-e-treinamento-dstaids-sp/homepage/ destaques/divulgacao-da-9-campanha-estadual-fique-sabendo-2016-testagem-de-hiv-e-sifilis

24. Marrazzo JM, Coffey P, Bingham A. Sexual practices, risk perception and knowledge of sexually transmitted disease risk among lesbian and bisexual women. Perspect Sex Reprod Health 2005; 37(1):6-12.
25. Lima MAS. Vulnerabilidade e prevenção às DST's nas práticas afetivo-sexuais de Lésbica [dissertação]. João Pessoa: Universidade Federal da Paraíba; 2016.

26. Brasil. Ministério da Saúde (MS). Portaria no 2.836, de 1 de dezembro de 2011. Institui, no âmbito do Sistema Único de Saúde (SUS), a Política Nacional de Saúde Integral de Lésbicas, Gays, Bissexuais, Travestis e Transexuais (Política Nacional de Saúde Integral LGBT). Diário Oficial da União 2011; 2 dez.

27. Mello APL. Apresentação da pesquisa: panorama da saúde de mulheres lésbicas e bissexuais: um olhar a partir do discurso de profissionais de saúde da família. In: Relatório da Oficina Atenção à Saúde de Mulheres Lésbicas e Bissexuais realizada em Brasília de 23 a 25 de abril de 2014. Brasília: MS; 2014. p. 19-23.

28. Brasil. Ministério da Saúde (MS). Política Nacional de Atenção Básica. Brasília: MS; 2012.

29. Dolan KD, Davis PW. Nuances and shifts in lesbian women's constructions of STI and HIV vulnerability. Soc Sci Med 2003; 57(1):25-38.

30. Muzny CA, Sunesara IR, Martin DH, Mena LA. Sexually transmitted infections and risk behaviors among African american women who have sex with women: does sex with men make a difference? Sex Transm Dis 2011; 38(12):1118-1125

Artigo apresentado em 02/11/2018

Aprovado em 22/02/2019

Versão final apresentada em 24/02/2019 
